\title{
Circumscribed Polygons of Small Area
}

\author{
Dan Ismailescu
}

Received: 8 October 2007 / Revised: 27 February 2008 / Published online: 28 March 2008

(C) Springer Science+Business Media, LLC 2008

Abstract Given any plane strictly convex region $K$ and any positive integer $n \geq 3$, there exists an inscribed $2 n$-gon $Q_{2 n}$ and a circumscribed $n$-gon $P_{n}$ such that

$$
\frac{\operatorname{Area}\left(P_{n}\right)}{\operatorname{Area}\left(Q_{2 n}\right)} \leq \sec \frac{\pi}{n} \text {. }
$$

The inequality is the best possible, as can be easily seen by letting $K$ be an ellipse. As a corollary, it follows that for any convex region $K$ and any $n \geq 3$, there exists a circumscribed $n$-gon $P_{n}$ such that

$$
\frac{\operatorname{Area}\left(P_{n}\right)}{\operatorname{Area}(K)} \leq \sec \frac{\pi}{n}
$$

This improves the existing bounds for $5 \leq n \leq 11$.

Keywords Convex regions · Circumscribed polygons

\section{Introduction}

Given a plane convex region $K$ and a positive integer $n \geq 3$, let $C_{n}(K)$ denote the circumscribed $n$-gon of minimum area and let $I_{n}(K)$ denote the inscribed $n$-gon of maximum area. Notice that both $C_{n}(K)$ and $I_{n}(K)$ may not necessarily be proper $n$-gons. For instance if $K$ is a triangle, then $C_{n}(K)=I_{n}(K)=K$ for every $n \geq 3$.

In order to avoid dealing with these special situations, in the following we will consider only bodies that are strictly convex, that is, their boundaries do not contain line segments. This restriction ensures that both $C_{n}(K)$ and $I_{n}(K)$ are proper convex

D. Ismailescu $(\varangle)$

Hofstra University, Hempstead, NY 11549-1000, USA

e-mail: Dan.P.Ismailescu@ hofstra.edu 


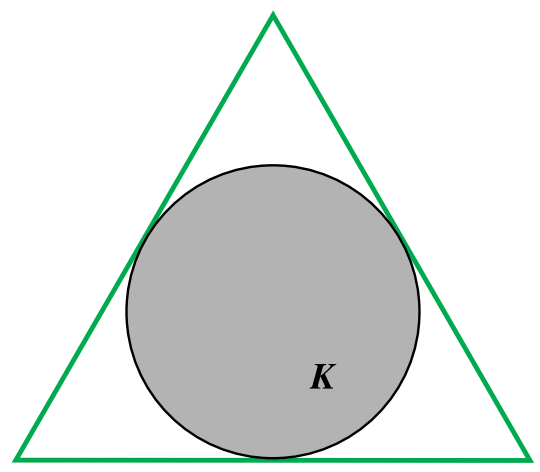

a)

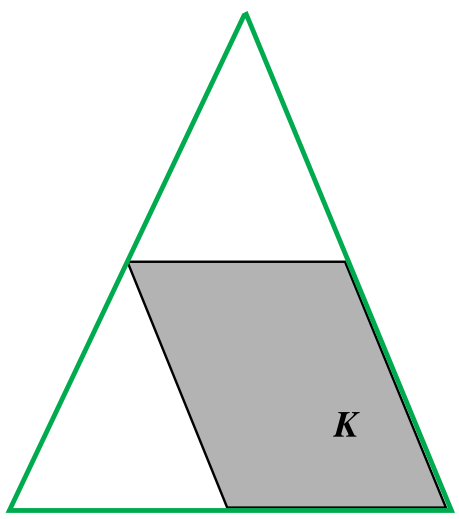

b)

Fig. 1 (a) $K=$ circle of unit area, Area $\left(C_{3}(K)\right)=\frac{3 \sqrt{3}}{\pi} \approx 1.65 \ldots$ (b) $K=$ parallelogram of unit area, $\operatorname{Area}\left(C_{3}(K)\right)=2$

$n$-gons. The results we obtain for the class of strictly convex bodies can then be extended to arbitrary convex bodies by a standard approximation argument.

The problems of finding $i_{n}:=\inf \operatorname{Area}\left(I_{n}(K)\right)$ and $c_{n}:=\sup \operatorname{Area}\left(C_{n}(K)\right)$ for a given integer $n \geq 3$ when $K$ ranges over all convex domains of unit area have been studied for their intrinsic interest as well as for their connection with packing and covering problems (see, e.g., [1, Sects. 22 and 27], [15], [13], [7, Chap. II, Sect. 4], and [2]).

It is well known that these quantities are actually attained, because the space of affine equivalence classes of convex regions is compact (see [12]). This motivates the problem of finding the convex regions $K$ that produce the extreme values $i_{n}$ and $c_{n}$.

In 1939, Sas [16] proved that

$$
i_{n}=\frac{n}{2 \pi} \sin \frac{2 \pi}{n},
$$

the infimum being reached if and only if $K$ is an ellipse.

One would expect a similar statement to hold for circumscribed polygons. If $K$ is an ellipse of unit area, it is easy to prove that the circumscribed $n$-gon of minimum area is affinely regular and its area is $\frac{n}{\pi} \tan \frac{\pi}{n}$. It would therefore be tempting to conjecture that $c_{n}=\frac{n}{\pi} \tan \frac{\pi}{n}$. However, one can readily check that this equality does not hold. It is sufficient to note that for $n=3$ the area of $C_{3}(K)$ equals 2 if $K$ is a parallelogram of unit area while it is only $\sqrt{27} / \pi=1.65 \ldots$ if $K$ is a circle (or an ellipse) of unit area; see Fig. 1.

It has been proven by many authors (see $[4,5,8])$ that $c_{3}=2$ and the extremal domains are the parallelograms. For $n \geq 4$ the extremal domains are not known and all we can realistically hope for is to obtain good bounds for $c_{n}$.

To illustrate how difficult it is to find the exact value of $c_{n}$ for $n \geq 4$, let us mention that the problem of finding $c_{6}$ is related to the still unsolved problem of determining the centrally symmetric convex region of lowest packing density. An old conjecture 
of Reinhardt [15] states that the extremal domain is the so-called "smoothed octagon", a convex region resulting from a regular octagon by cutting off each vertex $v_{i}$ with a hyperbolic arc from the hyperbola that is tangent to $v_{i} v_{i-1}$ and $v_{i} v_{i+1}$ and has the supporting lines $v_{i-1} v_{i-2}$ and $v_{i+1} v_{i+2}$ as asymptotes. Since by a result of Fejes Tóth (see [7]) the packing density of any centrally symmetric convex region $K$ equals the ratio Area $(K) / \operatorname{Area}\left(C_{6}(K)\right)$ solving Reinhardt's conjecture is equivalent to determining $c_{6}^{\prime}:=\sup A r e a\left(C_{6}(K)\right)$ when $K$ ranges over all centrally symmetric convex domains of unit area.

Modifying the averaging technique Sas employed to prove (1), Fejes Tóth [6] showed that

$$
c_{n} \leq \frac{n-2}{\pi} \tan \frac{\pi}{n-2} \quad \text { for every } n \geq 5 .
$$

This is currently the best asymptotic result. Fejes Tóth also proved the following theorem.

Theorem 1.1 ([7, Chap. II, Sect. 4]) Let $K$ be a convex domain whose boundary contains from a circle of perimeter $L$ two diametrically opposite arcs with total length $4 L / n$. Then one can circumscribe about $K$ an $n$-gon $U_{n}$ such that $\operatorname{Area}\left(U_{n}\right) \leq \operatorname{Area}(K) \frac{n}{\pi} \tan \frac{\pi}{n}$.

This suggests that for large values of $n$ the extremal domains are approximately ellipses. The problem of estimating $c_{n}$ is therefore more interesting for small values of $n$. Chakerian [4] proved that $c_{4} \leq \sqrt{2}$ (the equality sign being later removed by Kuperberg in [9]) and that

$$
c_{n} \leq \frac{2 \pi}{n} \csc \frac{2 \pi}{n} \quad \text { for every } n \geq 5
$$

Notice that Chakerian's estimate (3) is better that Fejes Tóth's bound (2) only for $n \leq 7$.

The main result of this paper is given by the following theorem.

Theorem 1.2 For every plane strictly convex region $K$ there exists an inscribed $2 n$ gon $Q_{2 n}$ and a circumscribed n-gon $P_{n}$ such that

$$
\frac{\operatorname{Area}\left(P_{n}\right)}{\operatorname{Area}\left(Q_{2 n}\right)} \leq \sec \frac{\pi}{n} \text {. }
$$

The inequality is the best possible, as can be seen by letting $K$ be an (unit area) ellipse. It can be shown that, in this case, both the circumscribed $n$-gon of minimum area and the inscribed $2 n$-gon of maximum area are affinely regular and their areas are $\frac{n}{\pi} \tan \frac{\pi}{n}$ and $\frac{n}{\pi} \sin \frac{\pi}{n}$, respectively.

It follows that the upper bound in Theorem 1.2 cannot be improved. Besides extending the known results $c_{3}=2$ and $c_{4} \leq \sqrt{2}$, Theorem 1.2 provides a new upper bound for $c_{n}$. 
Fig. 2 The inscribed $n$-gon $Q$ cuts off triangles of equal area from the circumscribed $n$-gon $P$. For each such pair inequality (4) holds

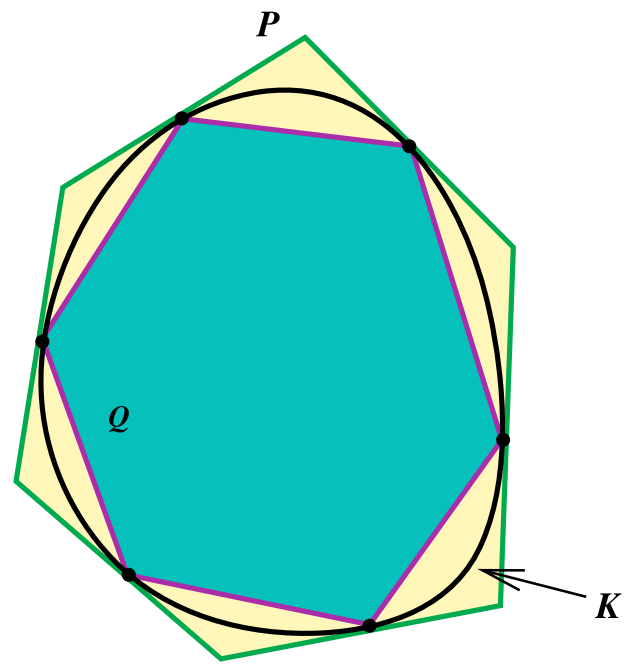

\section{Corollary 1.3}

$$
c_{n} \leq \sec \frac{\pi}{n} \quad \text { for every } n \geq 3 \text {. }
$$

This improves Chakerian's estimate (3) for every $n \geq 5$ and it is better than Fejes Tóth's bound (2) for all $5 \leq n \leq 11$.

\section{The Tools}

For the proof of Theorem 1.2 we are going to need two results. The first one is due to Lázár ([10], see also [7]).

Theorem 2.1 Given a plane strictly convex region $K$ and a positive integer $n \geq 3$, there exists a circumscribed convex $n$-gon $P$ such that

$$
\frac{\operatorname{Area}(P)}{\operatorname{Area}(Q)} \leq \sec ^{2} \frac{\pi}{n}
$$

where $Q$ is the $n$-gon spanned by the points where $P$ touches the boundary of $K$.

Lázár's original result is actually stronger. He proves that if the circumscribed $n$-gon $P$ has the property that the triangles cut off from $P$ by the sides of the $n$-gon $Q$ have the same area, then the above inequality holds (see Fig. 2).

The other result to be used later is a corollary of the Brunn-Minkowski inequality. This inequality is valid in a much more general setting than used here. We refer the interested reader to the original works of Brunn [3] and Minkowski [14]; for a modern treatment one should consult [17]. 
Fig. $3 \operatorname{Area}(Q, R)=$ $\frac{1}{2} \sum_{i=1}^{n} l_{i} h_{i}=$ Area of the shaded polygon

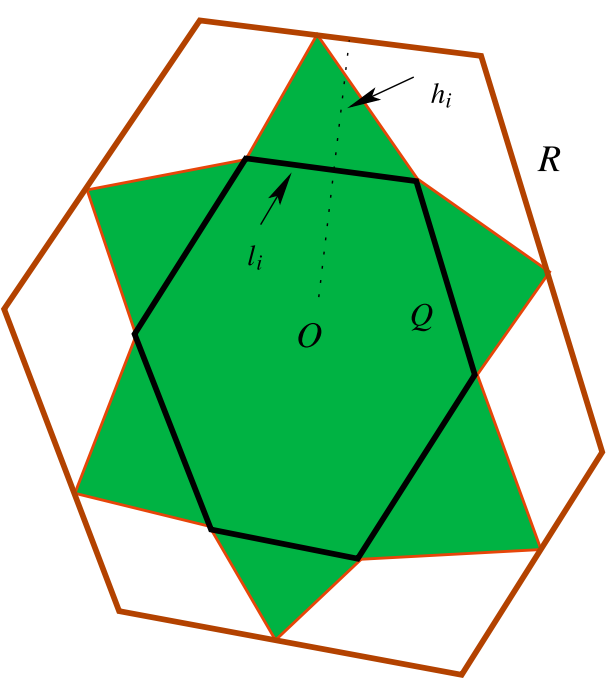

Given two convex regions $K$ and $L$, recall that $\operatorname{Area}(K, L)$, the mixed area of $K$ and $L$, is defined by the equality

$$
\operatorname{Area}(s K+(1-s) L)=s^{2} \operatorname{Area}(K)+2 s(1-s) \operatorname{Area}(K, L)+(1-s)^{2} \operatorname{Area}(L) .
$$

The Brunn-Minkowski inequality states that

$$
\operatorname{Area}(K, L) \geq \sqrt{\operatorname{Area}(K) \cdot \operatorname{Area}(L)} .
$$

Let $R$ and $Q$ be two convex $n$-gons whose corresponding sides are parallel (see Fig. 3). Then Area $(Q, R)$ can be easily described as follows. Let $O$ be a fixed point inside $Q$. If $l_{i}$ is the length of a side of $Q$, denote by $h_{i}$ the distance from $O$ to the corresponding side of $R$. Then

$$
\operatorname{Area}(Q, R)=\frac{1}{2} \sum_{i=1}^{n} l_{i} h_{i} .
$$

Lyusternik [11] presented an excellent introductory account of the properties of mixed areas as well as proofs of both (5) and (6).

\section{Proof of the Main Theorem}

Given $K$ a plane strictly convex body consider the following polygons:

$P:=$ a circumscribed $n$-gon with the property from Theorem 2.1 .

$Q:=$ the inscribed $n$-gon spanned by the points where $P$ touches $K$.

$R:=$ the circumscribed $n$-gon parallel to $Q$.

$S:=$ the inscribed $2 n$-gon spanned by the vertices of $Q$ and by the points where $R$ touches $K$; Fig. 4 illustrates the case $n=5$. 
Fig. 4 The polygons considered in the proof of the main theorem

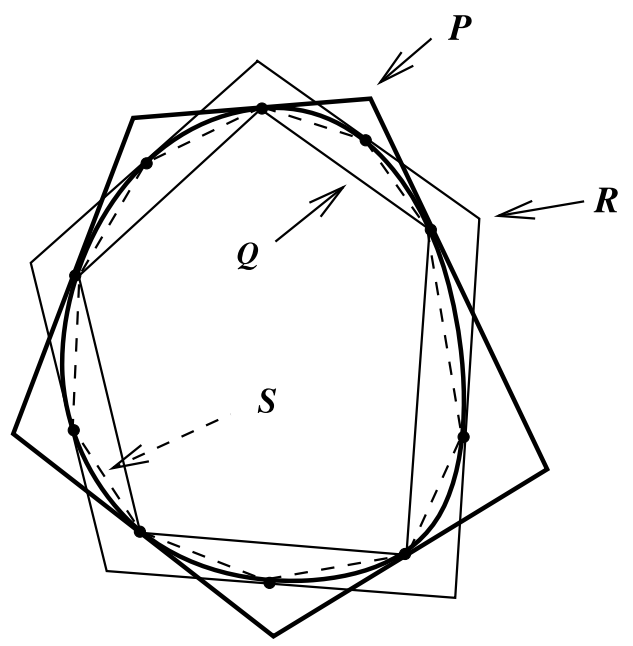

By our choice of $P$ we have that

$$
\operatorname{Area}(P) \leq \operatorname{Area}(Q) \cdot \sec ^{2} \frac{\pi}{n}
$$

On the other hand, by our previous argument it is easy to see that

$$
\operatorname{Area}(S)=\frac{1}{2} \sum_{i=1}^{n} l_{i} h_{i}=\operatorname{Area}(Q, R)
$$

which by using (5) gives

$$
\operatorname{Area}(S) \geq \sqrt{\operatorname{Area}(Q) \cdot \operatorname{Area}(R)} .
$$

From (7) and (8) we obtain that

$$
\frac{\min (\operatorname{Area}(P), \operatorname{Area}(R))}{\operatorname{Area}(S)} \leq \frac{\sqrt{\operatorname{Area}(P) \cdot \operatorname{Area}(R)}}{\operatorname{Area}(S)} \leq \frac{\sqrt{\operatorname{Area}(Q) \cdot \operatorname{Area}(R)} \cdot \sec \frac{\pi}{n}}{\operatorname{Area}(S)},
$$

that is,

$$
\frac{\min (\operatorname{Area}(P), \operatorname{Area}(R))}{\operatorname{Area}(S)} \leq \sec \frac{\pi}{n} .
$$

This proves the theorem.

\section{Conclusions and Open Problems}

In this paper we proved a new upper bound for $c_{n}$ which improves the existing bounds for small values of $n$. As mentioned in the introduction, the problem of determining 
the exact value of $c_{n}$ is extremely difficult. It is apparent from the reference list that the progress has been slow and sporadic.

The only known exact value is $c_{3}=2$. One may consider the situation when $n=4$ as it seems to be the easiest unsolved case. It is known that $c_{4}<\sqrt{2} \approx 1.414 \ldots$. On the other hand, Kuperberg [9] asked the following question:

Open Problem Is it true that the (affinely) regular pentagon is the extremal region for which $c_{4}$ is attained? In other words, is it true that $c_{4}=\frac{3}{\sqrt{5}} \approx 1.341 \ldots$ ?

While proving this may be difficult, it would be of interest if one can narrow the gap between the lower and upper bounds for $c_{4}$.

Acknowledgements The author thanks Gabor Fejes Tóth and Wlodzimierz Kuperberg for their helpful insights.

\section{References}

1. Blaschke, W.: Vorlesungen über Differentialgeometrie II. Affine Differentialgeometrie. Die Grundlehren der mathematischen Wissenschaften in Eizeldarstellungen Bd. 7. Springer, Berlin (1923)

2. Brass, P., Moser, W., Pach, J.: Research Problems in Discrete Geometry. Springer, New York (2005)

3. Brunn, H.: Über Ovale und Eiflächen. Inaugural Dissertation, München (1887)

4. Chakerian, G.D.: Minimum area of circumscribed polygons. Elem. Math. 28, 108-111 (1973)

5. Eggleston, H.G.: On triangles circumscribing plane convex sets. J. Lond. Math. Soc. 28, 36-46 (1953)

6. Fejes, L.: Eine Bemerkung zur Approximation durch $n$-Eckringe. Compos. Math. 7, 474-476 (1940)

7. Fejes Tóth, L.: Lagerungen in der Ebene, auf der Kugel und im Raum. Die Grundlehren der Mathematischen Wissenschaften in Einzeldarstellungen mit besonderer Berücksichtigung der Anwendungsgebiete, Band LXV. Springer, Berlin (1953). (German)

8. Gross, W.: Über affine Geometrie XIII: Eine Minimumeigenschaft der Ellipse und des Ellipsoids. Ber. Verh. Sächs. Akad. Wiss. Leipz., Math.-Nat.wiss. Kl. 70, 38-54 (1918)

9. Kuperberg, W.: On minimum area quadrilaterals and triangles circumscribed about convex plane regions. Elem. Math. 38(3), 57-61 (1983)

10. Lázár, D.: Sur l'approximation des courbes convexes par des polygones. Acta Univ. Szeged. Sect. Sci. Math. 11, 129-132 (1947). (French)

11. Lyusternik, L.A.: Convex Figures and Polyhedra. Heath, Boston (1966). Translated and adapted from the first Russian edition (1956) by Donald L. Barnett

12. Macbeath, A.M.: A compactness theorem for affine equivalence classes of convex regions. Can. J. Math. 3, 54-61 (1951)

13. Mahler, K.: On the minimum determinant and the circumscribed hexagons of a convex domain. Ned. Akad. Wet. 50, 692-703 (1947). Indag. Math. 9, 326-337 (1947)

14. Minkowski, H.: Geometrie der Zahlen. Teubner, Leipzig (1896)

15. Reinhardt, K.: Über die dichteste gitterförmige Lagerung kongruenter Bereiche in der Ebene und eine besondere Art konvexer Kurven. Abh. Math. Semin. Hamb. 10, 216-230 (1934)

16. Sas, E.: Über eine Extremumeigenschaft der Ellipsen. Compos. Math. 6, 468-470 (1939)

17. Schneider, R.: Convex Bodies: The Brunn-Minkowski Theory. Cambridge University Press, Cambridge (1993) 